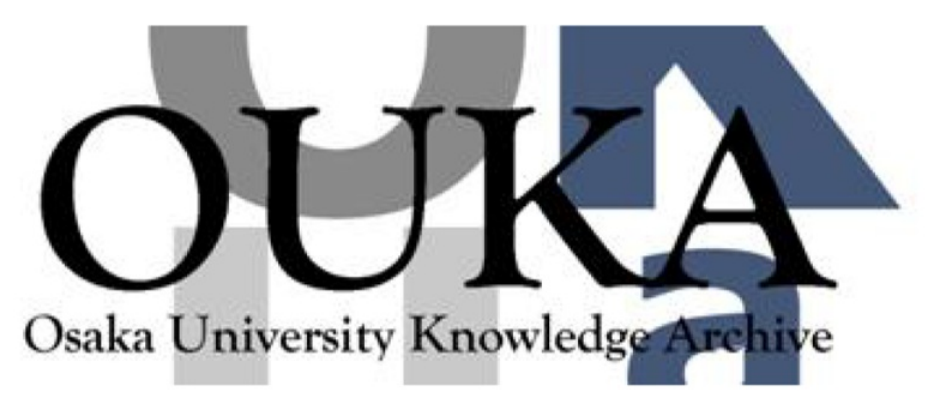

\begin{tabular}{|c|l|}
\hline Title & $\begin{array}{l}\text { Development of 170MHz electrodeless quartz- } \\
\text { crystal microbalance immunosensor with } \\
\text { nonspecifically immobi lized receptor proteins }\end{array}$ \\
\hline Author(s) & $\begin{array}{l}\text { Ogi, Hirotsugu; Nagai, Hironao; Fukunishi, Yuj i } \\
\text { et al. }\end{array}$ \\
\hline Citation & $\begin{array}{l}\text { Japanese Journal of Applied Physics. 49 } \\
\text { p.07HD07 }\end{array}$ \\
\hline Issue Date & $2010-07-20$ \\
\hline oaire:version & AM \\
\hline URL & https://hdl. handle.net/11094/84165 \\
\hline rights & \\
\hline Note & \\
\hline
\end{tabular}

Osaka University Knowledge Archive : OUKA

https://ir. Library. osaka-u. ac. jp/

Osaka University 


\title{
Development of $170 \mathrm{MHz}$ Electrodeless Quartz-Crystal Microbalance Immunosensor with Nonspecifically Immobilized Receptor Proteins
}

\author{
Hirotsugu OGI $^{*, 1,2}$, Hironao NAGAI ${ }^{1}$, Yuji FUKUNISHI ${ }^{1}$, Taiji YANAGIDA ${ }^{1}$, Masahiko \\ HIRAO $^{1}$, and Masayoshi NISHIYAMA ${ }^{3}$ \\ ${ }^{1}$ Graduate School of Engineering Science, Osaka University, 1-3 Machikaneyama, Toyonaka, Osaka \\ 560-8531, Japan \\ ${ }^{2}$ PRESTO, JST, 4-1-8 Honcho, Kawaguchi, Saitama, 102-0075 Japan \\ ${ }^{3}$ Renovation Center of Instruments for Science Education and Technology, Osaka University, 1-2 \\ Machikaneyama, Toyonaka, Osaka 560-0043, Japan
}

Staphylococcus aureus protein A (SPA) shows high nonspecific binding affinity on a naked quartz surface, and it can be used as the receptor protein for detecting immunoglobulin $G(\operatorname{IgG})$, the most important immunoglobulin. The immunosensor ability, however, significantly depends on the immobilization procedure. In this work, the effect of the nonspecific immobilization procedure on the sensor sensitivity is studied using a home-built electrodeless quartz-crystal microbalance (QCM) biosensor. The pure-shear vibration of a 9.7- $\mu \mathrm{m}$-thick AT-cut quartz plate is excited and detected in liquids by the line antenna located outside the flow channel. SPA molecules are immobilized on the quartz surfaces, and human IgG is injected to monitor the binding reaction between SPA and IgG. This study reveals that a long (nearly $24 \mathrm{~h}$ ) immersion procedure is required for immobilizing SPA to achieve the tight biding with the quartz surfaces.

\section{Introduction}

Quartz-crystal microbalance (QCM) biosensor is a label-free biosensor, allowing the realtime monitoring of binding reactions between biomolecules through the resonance frequency of the quartz oscillator. The receptor proteins are immobilized on the surfaces of the quartz oscillator, and the target analyte is injected in the solution where the oscillator is located. Adsorption of the target protein on the receptor protein causes an increase in the effective mass, leading to the decrease in the resonance frequency of the oscillator. ${ }^{1,2)}$ The viscosity effect $^{3)}$ and coupled-water mass ${ }^{4)}$ also contribute to the decrease in the resonance frequency, making the quantitative analysis complicated.

The mass sensitivity of the QCM significantly increases as the oscillator mass decreases. Because reduction of the sensing area causes reduction of the amount of adsorbed proteins, the oscillator-mass reduction has to be achieved by decreasing the thickness of the oscillator: A high-frequency QCM biosensor is therefore required for highly sensitive detection of a target analyte. The high-frequency QCM biosensor is also required for quantitative analysis, because the viscosity effect becomes insignificant compared with the mass loading effect at 
high frequencies. ${ }^{5)}$ Thus, intensive efforts have been made to develop high-frequency QCM biosensors. ${ }^{6-9)}$ Recently, we have developed a $170 \mathrm{MHz}$ QCM biosensor, ${ }^{10)}$ which showed a much higher mass sensitivity for the detection of human immunoglobulin $\mathrm{G}$ ( $\mathrm{hIgG}$ ) than a conventional $5 \mathrm{MHz}$ QCM biosensor by three orders of magnitude.

Here, we show a biosensor system using nonspecific adsorption of receptor proteins on the quartz surfaces. Many biosensor chips used linker materials, such as a self-assembled monolayer (SAM), between receptor proteins and the sensor surface. Gold films have been deposited on the sensor surfaces in this case, because gold exhibits high binding affinity to thiol-terminated molecules like a SAM, on which any receptor protein can be immobilized. However, gold thin films on quartz surfaces significantly deteriorate the QCM sensitivity because of the much larger mass density of gold than quartz. ${ }^{10)}$ The linker-free immobilization of receptor proteins on the quartz surfaces also contributes to shorten the assay time. Thus, the immobilization of receptor proteins on quartz surfaces is an important task for immunosensors.

We have recently suggested that staphylococcus aureus protein A (SPA) shows high binding affinity with naked quartz surfaces, ${ }^{11)}$ and that it can be used as a receptor protein for detecting hIgG. In this study, we develop a $170 \mathrm{MHz}$ electrodeless QCM immunosensor with nonspecifically immobilized SPA proteins for detecting hIgG molecules, and discuss the effect of the immobilization procedure on the sensing ability.

\section{Experimental Procedure}

We mechanically polished an AT-cut quartz plate to obtain a 9.7- $\mu$ m-thick blank QCM sensor chip. (The $R_{a}$ value for the surface roughness was smaller than $0.5 \mathrm{~nm}$.) Part of the quartz oscillator was lightly sandwiched by $1 \mathrm{~mm}$ silicon rubber gaskets, and the oscillator was set in the sensor cell, as shown in Fig. 1. (As shown later, we immobilized SPA on the quartz surfaces before or after setting the sensor chip in the sensor cell.) The sensor cell was installed in a temperature-controlled box, where the solution temperature was maintained at $37^{\circ} \mathrm{C}$. The home-built flow-injection system ${ }^{8)}$ was used for monitoring the frequency change during the binding reaction between SPA and hIgG. (The high-affinity binding between them was well confirmed. ${ }^{12,13)}$ ) The carrier solution was a phosphate-buffer solution (PBS) of $\mathrm{pH}$ 7.4 , and the flow rate was fixed at $500 \mu \mathrm{L} / \mathrm{min}$.

Two copper wires were used as the line antennas, and a thin copper film was located outside the flow channel as the grounding. Radio-frequency tone bursts were applied to the generation wire to provide a quasistatic electric field along the thickness direction of the crystal. After the excitation, the detection wire receives the reverberation signals of the crystal, which were fed to the superheterodyne spectrometer. The phase and amplitude were extracted from the received signal to determine the resonance-frequency change. ${ }^{8)}$

We first immobilized SPA molecules on the quartz surfaces using a flow-injection system. The quartz oscillator was first cleaned in a piranha solution $\left(98 \% \mathrm{H}_{2} \mathrm{SO}_{4}: 33 \% \mathrm{H}_{2} \mathrm{O}_{2}=7: 3\right)$ for 
$1 \mathrm{~h}$ and rinsed with ultrapure water several times. It was set in the sensor cell, and the PBS solution flowed. After the resonance frequency became stable, a $4.7 \mu \mathrm{M}$ SPA/PBS solution was injected, which was followed by injection of the hIgG/PBS solution.

We next performed a long immersion procedure to make the nonspecific immobilization between SPA and quartz tighter. After cleaning with the piranha solution and rinsing with ultrapure water, the quartz oscillator was immersed in the SPA/PBS solution for $24 \mathrm{~h}$ at $4{ }^{\circ} \mathrm{C}$, and then rinsed with PBS several times and set in the sensor cell. The hIgG solutions were then injected. hIgG was obtained from Athens Research and Technology, Inc. (product no. 16-16-090707; purity 95\%), and SPA was obtained from Zymed Laboratories, Inc. (product no. 10-1100; purity $\sim 98 \%$ ).

\section{Results and Discussion}

Figure 2 shows the frequency response for the injection sequence of the SPA and hIgG solutions. When the SPA solution arrives at the sensor cell, the resonance frequency exponentially decreases, and then it exponentially increases when the solution is changed to PBS. These frequency behaviors correspond to the adsorption and dissociation of SPA molecules at the quartz surfaces. The exponential frequency changes strongly indicate that the nonspecific association and dissociation reactions can be explained by the thermodynamic reaction expressed by

$$
A+B \underset{k_{d}}{\stackrel{k_{a}}{\rightleftarrows}} A B
$$

Where $A$ and $B$ denote the SPA and the binding site on the quartz surface, respectively, and $A B$ denotes their complex. $k_{a}$ and $k_{d}$ are the reaction velocity constants for association and dissociation reactions, respectively. Considering the constant concentration of the SPA in the flowing solution during the reactions, the frequency changes during the association and dissociation reactions are expressed by ${ }^{14)}$

$$
\begin{array}{r}
\Delta f(t)=A_{1}\left\{\mathrm{e}^{-\left(k_{a} C_{A}+k_{d}\right) t}-1\right\}, \\
\Delta f(t)=-A_{2}\left\{\mathrm{e}^{-k_{d} t}-1\right\},
\end{array}
$$

respectively. Here, $t$ is time, $C_{A}$ is the concentration of the analyte in the solution, and $A_{1}$ and $A_{2}$ are positive constants. Thus, by fitting the exponential functions to the measurements (red and blue lines in Fig. 2), we determined $k_{a}$ and $k_{d}$ values, and the equilibrium constant $K_{A}=k_{a} / k_{d}$. They are shown in Table I along with those for other nonspecific binding pairs (IgG-sulfamethazine and IgG-histidine) and a specific binding pair between IgG and SPA.

The SPA-quartz affinity ( $K_{A}$ value) is significantly larger than those of the other nonspecific binding reactions, confirming the usefulness of SPA as the nonspecifically immobilized receptor protein for immunosensors. However, compared with the specific binding reaction, the SPA-quartz bond shows a lower $k_{a}$ value, meaning that SPA molecules require a longer 
time to be tightly immobilized on the quartz surface, and a short immobilization procedure would be insufficient and cause dissociation of SPA molecules from the quartz surface. Figure 2 shows this explicitly, where the frequency increases after the injection of PBS. Furthermore, subsequent injection of the hIgG solution $(t=24400 \mathrm{~s})$ causes a nonexponential decrease in the frequency. This is an unusual change in the specific binding reaction, because the frequency change has been well expressed by eq. (2) for the SPA-IgG binding reactions. ${ }^{16)}$ Thus, the dissociation reaction of SPA from the quartz surface and the binding reaction between SPA and IgG simultaneously proceed, leading to the non-exponential frequency change.

Therefore, we immobilized the SPA molecules by immersing the sensor chip in the $\mathrm{SPA} / \mathrm{PBS}$ solution for $24 \mathrm{~h}$. In this case, the frequency largely decreases in an exponential manner, as shown in Fig. 3, indicating insignificant dissociation of SPA from the surface. The amount of frequency change caused by the adsorption of hIgG molecules increased by a factor of 6 .

Figure 4 shows the frequency responses for the consecutive injection of the various concentration hIgG solutions. The exponential coefficient increases with the increase in the concentration, as predicted by eq. (2). Thus, by plotting the concentration and exponential coefficient, we determined the thermodynamic constants between hIgG and SPA, ${ }^{17)}$ which are shown in Table I. Their values are comparable with those measured by the SPA immobilized using a SAM, ${ }^{5)}$ indicating that the nonspecifically immobilized SPA works as a receptor layer.

\section{Conclusions}

A $170 \mathrm{MHz}$ electrodeless QCM immunosensor was developed, using nonspecifically adsorbed receptor proteins to detect IgG molecules in liquids. When the SPA molecules were immobilized by the flow-injection procedure, they were dissociated from the sensor surface when the carrier PBS flowed. The thermodynamic constants between SPA and the quartz surface were determined, and the association reaction velocity constant was found to be smaller than that in the specific binding cases. Thus, the long immobilization procedure was performed to make tight bonds between SPA and quartz surface, which showed highly sensitive detection of hIgG.

\section{Acknowledgement}

This study was partially supported by Life Phenomena and Measurement Analysis, PRESTO, by Japan Science and Technology Agency. 


\section{References}

1) G. Sauerbrey: Z. Phys. 155 (1959) 206 [in German].

2) H. Muramatsu, M. Dicks, E. Tamiya, and I. Karube: Anal. Chem. 59 (1987) 2760.

3) S. J. Martin, V. E. Granstaff, and G. C. Frye: Anal. Chem. 63 (1991) 2272.

4) P. Bingen, G. Wang, N. F. Steinmetz, M. Rodahl, and R. P. Richter: Anal. Chem. 80 (2008) 8880.

5) H. Ogi, K. Motohisa, T. Matsumoto, K. Hatanaka, and M. Hirao: Anal. Chem. 78 (2006) 6903.

6) M. Natesan, M. A. Cooper, J. P. Tran, V. R. Rivera, and M. A. Poli: Anal. Chem. 81 (2009) 3896.

7) H. Furusawa, M. Komatsu, and Y. Okahata: Anal. Chem. 81 (2009) 1841.

8) H. Ogi, T. Omori, K. Hatanaka, M. Hirao, and M. Nishiyama: Jpn. J. Appl. Phys. 47 (2008) 4021.

9) H. Ogi, K. Hatanaka, Y. Fukunishi, H. Nagai, M. Hirao, and M. Nishiyama: Jpn. J. Appl. Phys. 48 (2009) 07GF01.

10) H. Ogi, H. Nagai,Y. Fukunishi, M. Hirao, and M. Nishiyama: Anal. Chem. 81 (2009) 8068.

11) H. Ogi, Y. Fukunishi, H. Nagai, K. Okamoto, M. Hirao, and M. Nishiyama: Biosens. Bioelectron. 24 (2009) 3148.

12) G. Kronvall, P. G. Quie, and R. C. Williams Jr.: J. Immunol. 104 (1970) 273.

13) D. C. Hanson and V. N. Schumaker: J. Immunol. 132 (1984) 1397.

14) Y. Liu, X. Yu, R. Zhao, D. Shangguan, Z. Bo, and G. Liu: Biosens. Bioelectron. 19 (2003) 9.

15) Y. Liu, X. Yu, R. Zhao, D. Shangguan, Z. Bo, and G. Liu: Biosens. Bioelectron. 18 (2003) 1419 .

16) H. Ogi, K. Motohisa, K. Hatanaka, T. Ohmori, M. Hirao, and M. Nishiyama: Biosens. Bioelectron. 22 (2007) 3238.

17) H. Ogi, Y. Fukunishi, T. Omori, K. Hatanaka, M. Hirao, and M. Nishiyama: Anal. Chem. 80 (2008) 5494. 


\section{Tables}

Table I. Association and dissociation reaction velocity constants ( $k_{a}$ and $k_{d}$, respectively), and the equilibrium constant $K_{A}$ for nonspecific (NS) and specific (SP) binding pairs.

\begin{tabular}{ccccc}
\hline \hline & Pair & $k_{a}\left(10^{3} \mathrm{M}^{-1} \mathrm{~s}^{-1}\right)$ & $k_{d}\left(10^{-4} \mathrm{~s}^{-1}\right)$ & $K_{A}\left(10^{6} \mathrm{M}^{-1}\right)$ \\
\hline & SPA-quartz (present) & 0.48 & 3.1 & 1.6 \\
$\mathrm{NS}$ & IgG-sulfamethazine $^{14)}$ & 0.433 & 7.92 & 0.548 \\
$\mathrm{NS}$ & IgG-histidine $^{15)}$ & 0.324 & 0.011 & 0.0292 \\
\hline $\mathrm{SP}$ & SPA-IgG (present) $_{\mathrm{SP}}$ & 18 & 3.0 & 60 \\
$\mathrm{SPA}-\operatorname{IgG}$ & & 23.7 & 4.56 & 52.1 \\
\hline \hline
\end{tabular}




\section{Figure Captions}

Fig. 1 Cross-section of the sensor cell for the $170 \mathrm{MHz}$ electrodeless QCM. The solution flows along both surfaces of the quartz plate. Two line antennas and the grounding thin film are located outside the flow channel.

Fig. 2 (Color) Frequency response for the injection of the SPA and hIgG solutions. Red and blue lines are fitted exponential functions [eqs. (2) and (3), respectively], corresponding to the association and dissociation reaction-rate constants between SPA and the quartz surface, respectively. Arrows indicate the arrival times of solutions at the sensor cell.

Fig. 3 Comparison of the frequency response to the hIgG solution using the SPA immobilized by the flow-injection procedure (flow) with that by the immersion procedure for $24 \mathrm{~h}$ (immersion). The former caused a nonexponential frequency change, indicating dissociation of SPA from the quartz surface during the binding reaction between SPA and hIgG.

Fig. 4 Frequency response for the injection sequence of the hIgG solutions with various concentrations detected by the SPA immobilized by the immersion procedure. 


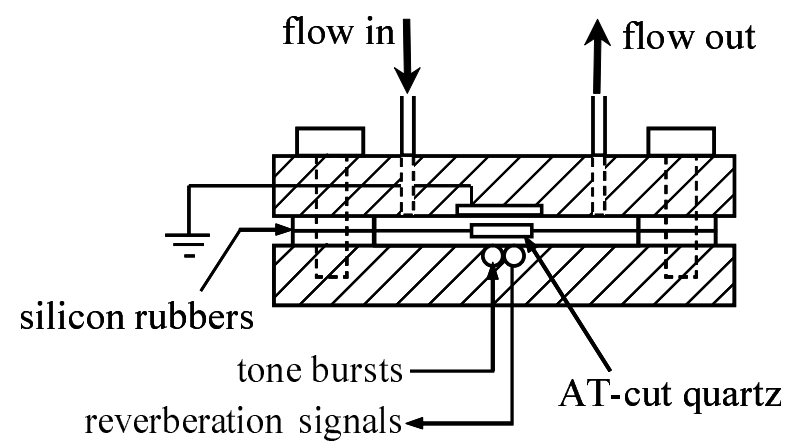

Fig. 1. 
Jpn. J. Appl. Phys.

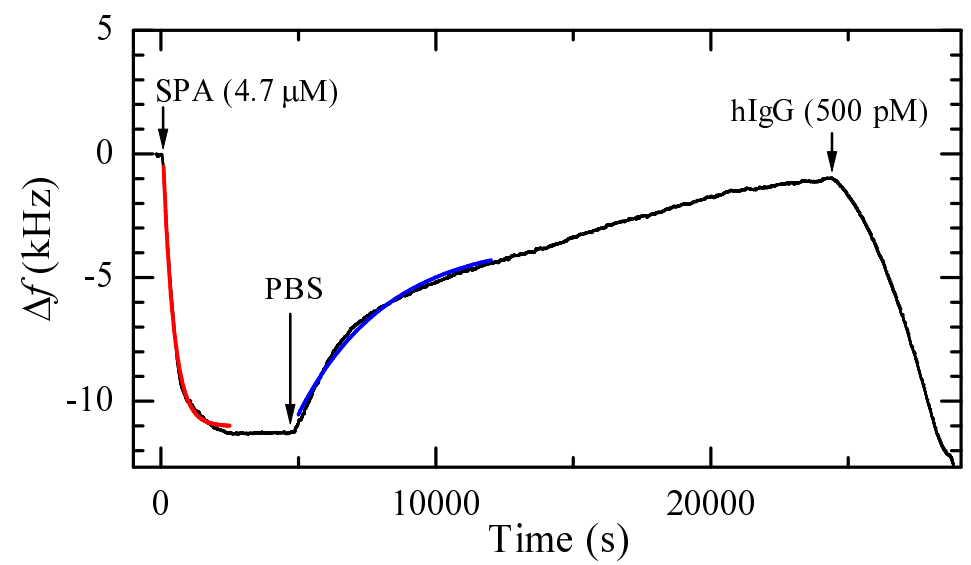

Fig. 2. 


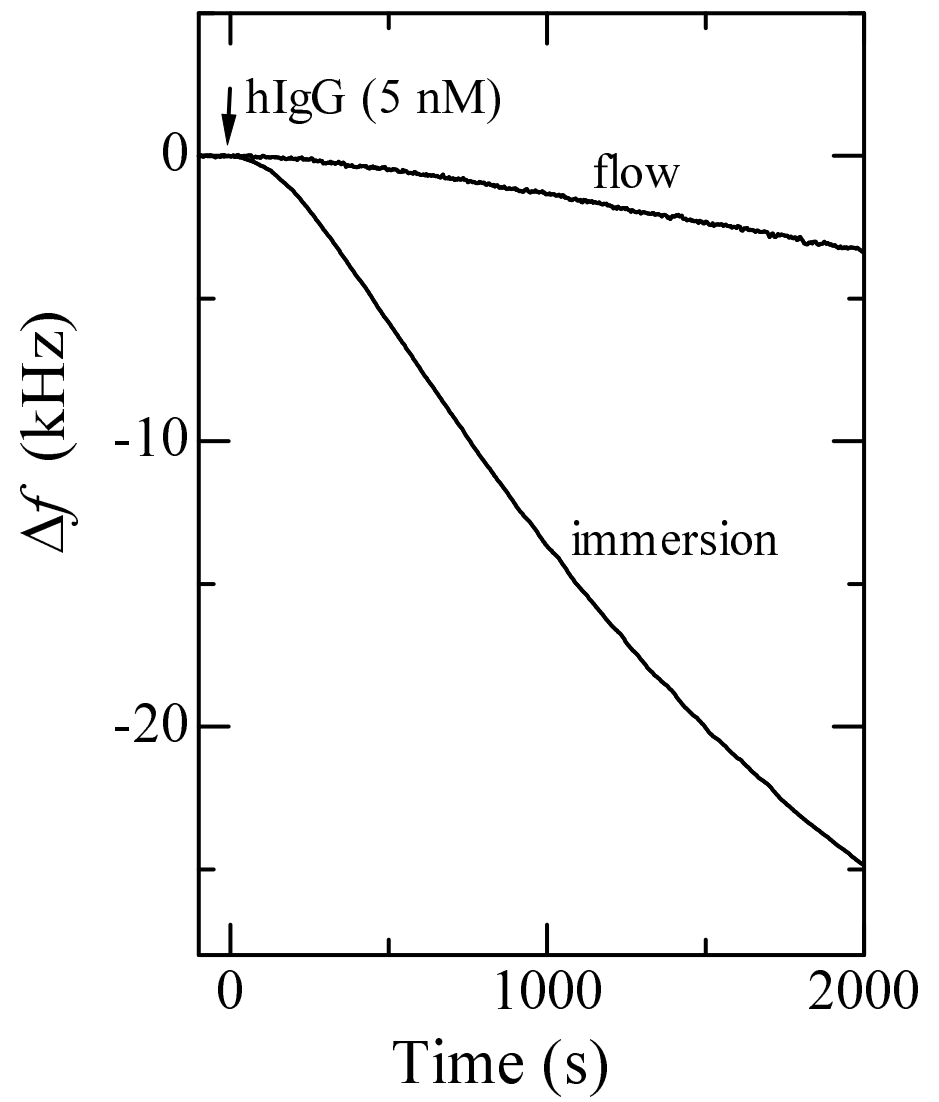

Fig. 3. 


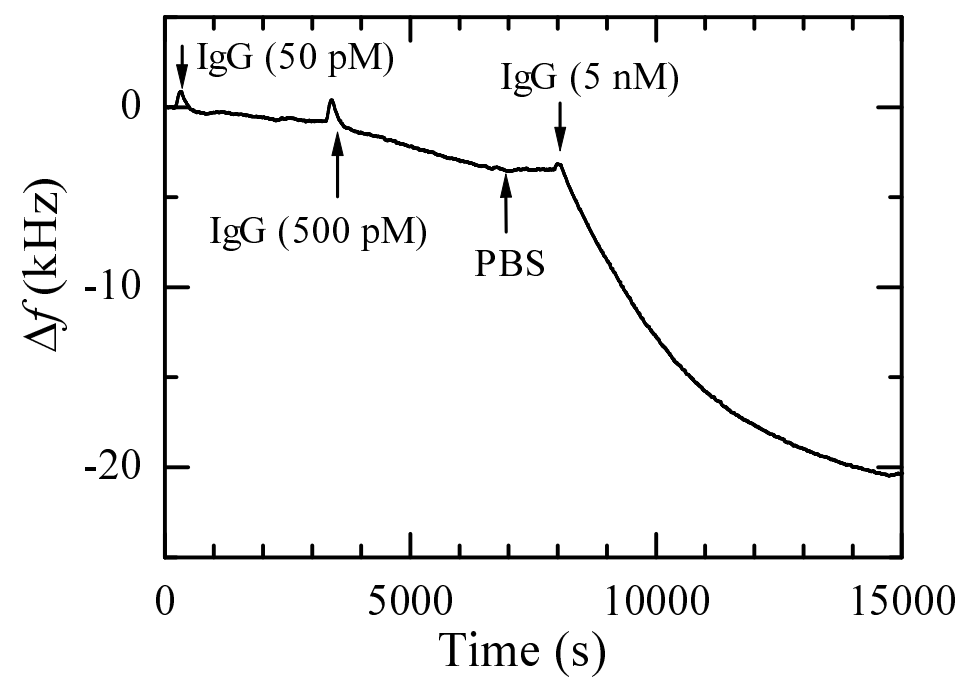

Fig. 4. 